$\xi=-1$

\title{
Securing Underwater Wireless Communication Networks
}

\author{
D. Naresh Kumar ${ }^{1 *}$ \\ ${ }^{l}$ m.Tech Scholar, Communication Systems,Sanketika Vidya Parishad Engineering College (Au), Visakhapatnam, Andhrapradesh, India. \\ *Corresponding Author E-Mail: D.Naresh37@Gmail.Com
}

\begin{abstract}
Underwater remote correspondence systems (UWCNs) are especially defenseless against vindictive assaults because of the high piece blunder rates, huge and variable proliferation deferrals, and low data transmission of acoustic channels. The special attributes of the Underwater acoustic correspondence channel and the contrasts between Underwater sensor systems and their ground-based partners require the improvement of productive and solid security instruments. In this class, an entire review of security for UWCNs is exhibited, and the exploration challenge for secure correspondence in this condition is sketched out.

UWCNs incorporate sensors and self-ruling Underwater vehicles (AUVs) that collaborate to perform particular applications, for example, Underwater observing. Coordination and data sharing amongst sensors and AUVs make the arrangement of security testing.
\end{abstract}

Keywords: UWCN's, Sensors, Synchronization, Security, Attacks, Underwater communication, Wireless sensor networks.

\section{Introduction}

Underwater remote correspondence systems (UWCNs) are constituted by sensors and self-ruling Underwater vehicles (AUVs) that associate to perform particular applications, for example, Underwater checking. Coordination and sharing of data amongst sensors and AUVs make the arrangement of security testing. The amphibian condition is especially problematic to malignant assaults because of the high piece blunder rates, vast and variable. spread postponements, and low transfer speed of acoustic channels.

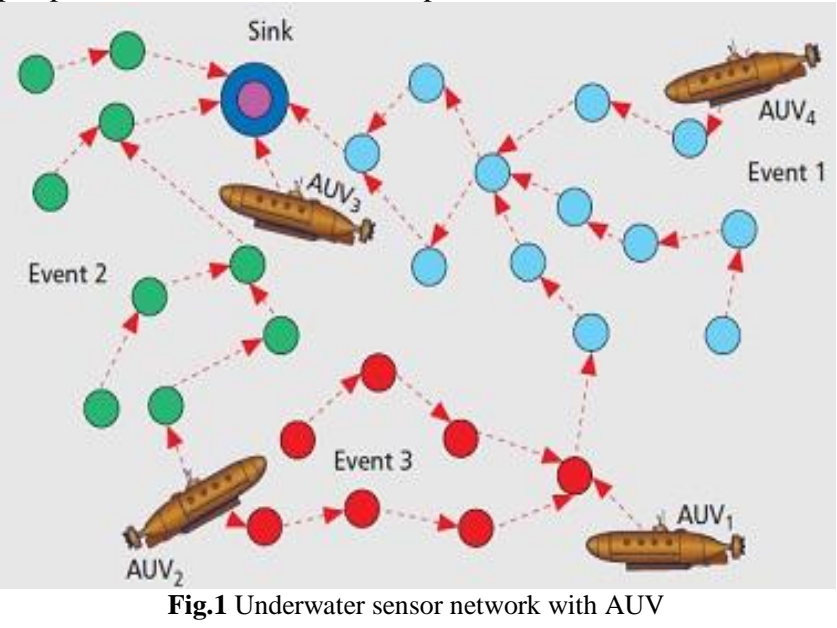

The oceanic condition is especially powerless against pernicious assaults because of the high piece mistake rates, vast and variable engendering delay, low transmission capacity of acoustic diverts in water. Accomplishing solid bury vehicle and sensor-AUV correspondence is particularly troublesome because of the versatility of AUVs and the development of sensors with water streams.

The exceptional attributes of the Underwater acoustic channel and the contrasts between such systems and their ground based part- ners require the advancement of proficient and dependable security instruments.

By sending a circulated and adaptable sensor arrange in a 3dimensional Underwater space, each Underwater sensor can screen and recognize ecological parameters and occasions locally. Thus, contrasted and remote detecting, UWCNs give a superior detecting and reconnaissance innovation to secure better information to comprehend the spatial and worldly complexities of Underwater situations. Display Underwater correspondence frameworks include the transmission of data as sound, electromagnetic (EM), or optical waves. Every one of these strategies has favorable circumstances and confinements.

\section{OVERVIEW (Underwater Wireless Communication Networks)}

Underwater remote correspondence systems (UWCNs) comprise of sensors and self-governing Underwater vehicles (AUVs) that connect, arrange and share data with each other to do detecting and checking capacities. A pictorial portrayal is demonstrated as follows:

In most recent quite a while, Underwater correspondence arrange (UWCN) has discovered an expanding use in a far reaching scope of uses, for example, beach front observation frameworks, ecological research, self-sufficient Underwater vehicle (AUV) activity, oil-fix support, gathering of information for water checking, connecting submarines to arrive, to give some examples.

The previously mentioned attributes of UWCNs have a few security issues related like bundle mistakes, listening in, change of parcels, and some more. Additionally since control utilization in Underwater interchanges is higher than in earthly radio correspondences vitality depletion assaults can diminish arrange life. 
The diverse assaults conceivable are Jamming, Wormholes, Selective Forwarding, Sybil Attacks, and so forth. Safeguards for these are talked about. Sticking can be overwhelmed by Spread Spectrum strategies, Wormhole identification is finished with a visual displaying utilizing Dis-VoW and different assaults can be countered by confirmation, check, and situating.

Open research challenges for secure restriction, steering and time synchronization are said.

In this paper UWCNs is talked about, with accentuation on the conceivable assaults, countermeasures and further openings and extension for advancement toward this path to enhance security of such systems.

\section{ATTACKS: UWCNs and Counter Measures Jamming}

A sticking assault comprises of meddling with the physical channel by setting up bearers on the frequencies neighbor hubs use to impart. Since Underwater acoustic recurrence groups are restricted, UWCNs are defenseless against narrowband sticking. Limitation is influenced by the replay assault when the assailant sticks the correspondence between a sender and a beneficiary, and later replays a similar message with stale data acting like the sender.

Spread range is the most well-known protection against sticking. Recurrence bouncing spread range (FHSS) and direct arrangement spread range (DSSS) in Underwater interchanges are drawing consideration for their great execution under clamor and multipath obstruction. These plans are impervious to obstruction from assailants, in spite of the fact that not trustworthy. An assailant can stick a wide band of the range or take after the exact bouncing grouping when a FHSS conspire is utilized. A high-control wideband sticking sign can be utilized to assault a DSSS plot. Underwater sensors under a sticking assault should attempt to save their energy. When sticking is consistent, sensors can change to rest mode and wake up occasionally to check if the assault is finished. When sticking is irregular, sensors can cradle information bundles and just send high-control high need messages to report the assault when a hole in sticking happens. In ground-based sensor systems, different sensors situated along the edge of the region under typical foundation commotion and report interruption to outside hubs. That will make any further movement be rerouted around the stuck area. In any case, this arrangement can't be connected to UWCNs, since hubs Underwater are generally meagerly conveyed, which implies there would not be sufficient sensors to delimit the stuck locale precisely and reroute movement around it.

\subsection{Wormhole Attack}

A wormhole is an out-of-band association made by the foe between two physical areas in a system with bring down deferral and higher transfer speed than customary associations. This association utilizes quick radio (over the ocean surface) or wired connects to essentially diminish the proliferation delay.

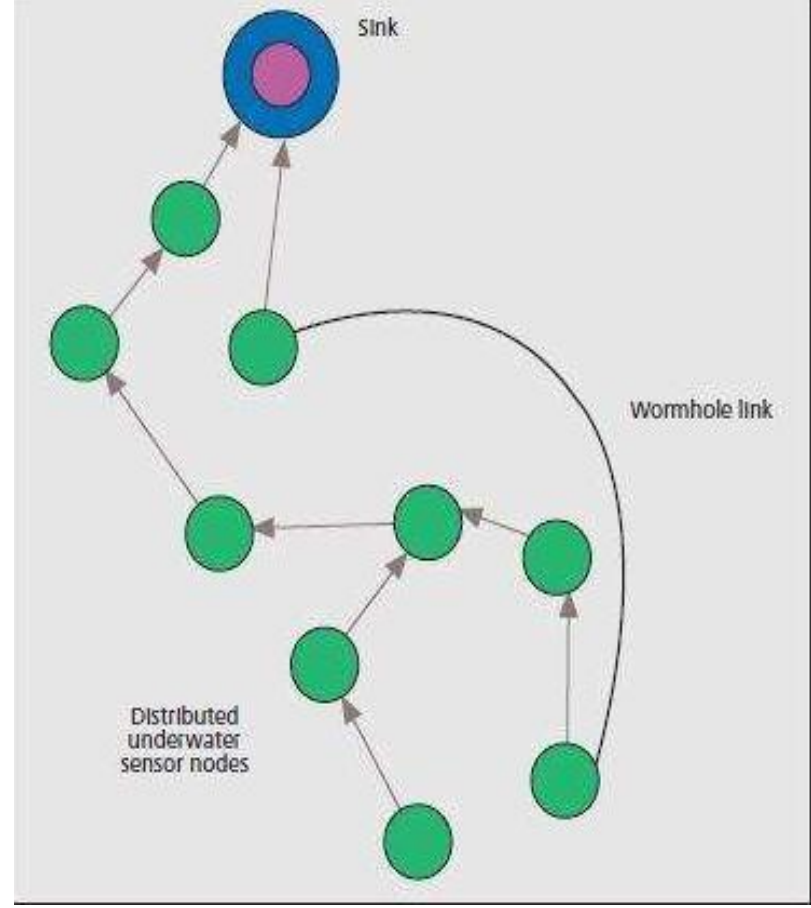

Fig.2: Underwater network with a wormhole link

\subsection{Sybil Attack}

An aggressor with numerous characters can put on a show to be in numerous spots on the double. Geographic steering conventions are additionally deceived on the grounds that a foe with various characters can claim to be in numerous spots without a moment's delay Authentication and position confirmation are techniques against this assault, despite the fact that position check in UWCNs is hazardous because of versatility.
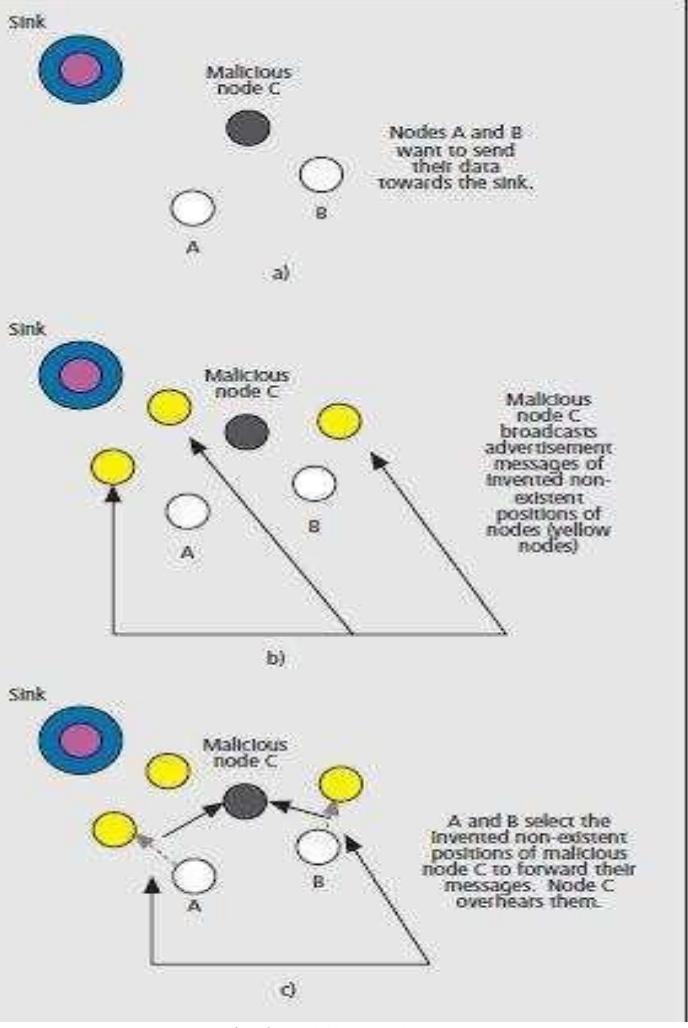

Fig.3 Sybil attack

\subsection{Sinkhole Attack}

In a sinkhole assault, a malignant hub endeavors to pull in rush hour gridlock from a specific territory toward it; for instance, the 
noxious hub can report a great course. Geographic directing and verification of hubs trading steering data are conceivable safeguards against this assault, yet geographic steering is as yet an open research subject in UWCNs.

\subsection{Hello Flood Attack}

A hub getting a HELLO bundle from a vindictive hub may translate that the foe is a neighbor; this supposition is false if the enemy utilizes high power for transmission. Bidirectional connection check can help secure against this assault, in spite of the fact that it isn't exact because of hub versatility and the high engendering deferrals of UWCNs. Verification is likewise a conceivable safeguard.

\subsection{Acknowledgment Spoofing}

A noxious hub catching bundles sent to neighbor hubs make this data parody connect layer affirmations with the goal of driving a feeble connection or a connection situated in a shadow zone. Shadow zones are framed when the acoustic beams are twisted and sound waves can't infiltrate. They cause high piece blunder rates and loss of availability. Along these lines, the directing plan controlled. An answer for this assault would be encryption of all parcels sent trough the system.

\subsection{Jamming Attack}

A sticking assault comprises of meddling with the physical channel by setting up bearers on the frequencies utilized by hubs to impart. Since it requires a considerable measure of vitality, aggressors for the most part assault in sporadic blasts. Since Underwater acoustic recurrence groups are thin (from a couple to many kilohertz), UWCNs are defenseless against narrowband sticking. Confinement is influenced by the replay assault when the aggressor sticks the correspondence between a sender and a collector, and later replays a similar message with stale data (an off base reference) acting like the sender.

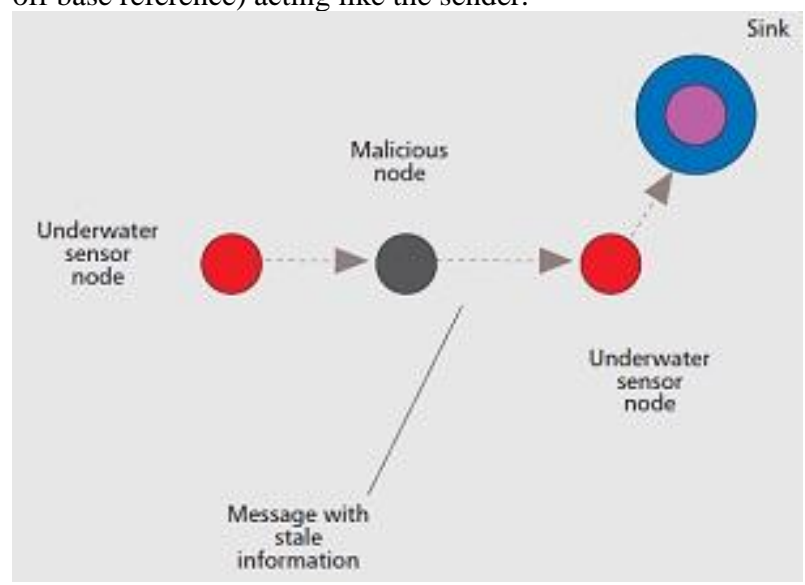

Fig.4: Jamming attack

In recurrence bouncing, a gadget transmits a flag on a recurrence for a brief timeframe, changes to an alternate recurrence and rehashes. The transmitter and beneficiary must be facilitated. Coordinate succession spreads the flag over a wide band, utilizing a pseudo-irregular piece stream. A beneficiary must know the spreading code to recognize the flag from clamor.

Recurrence bouncing plans are to some degree impervious to impedance from an aggressor who does not know the jumping succession. In any case, the assailant might have the capacity to stick a wide band of the range, or even take after the jumping arrangement by examining for the following transmission and rapidly tuning the transmitter.

\section{Security Requirements}

In UWCNs the following security requirements should be considered:

\subsection{Authentication}

Validation is the confirmation that the information was sent by a genuine sender. It is basic in military and security basic uses of UWCNs. Validation and key foundation are emphatically related in light of the fact that once at least two substances check each other's legitimacy, they can build up at least one mystery keys over the open acoustic channel to trade data safely; on the other hand, an effectively settled key can be utilized to perform verification. Conventional answers for key age and refresh (restoration) calculations ought to be adjusted to better address the attributes of the Underwater channel. A key age framework is suggested that requires just an edge identifier, lightweight calculation, and correspondence costs.

\subsection{Confidentiality}

Secrecy implies that data isn't open to unapproved outsiders. In this way, privacy in basic applications, for example, oceanic observation ought to be guaranteed Integrity: It guarantees that data has not been changed by any enemy. Numerous Underwater sensor applications for natural safeguarding, for example, water quality observing, depend on the trustworthiness of data.

\subsection{Availability}

The information ought to be accessible when required by an approved client. Absence of accessibility because of refusal ofbenefit assaults would particularly influence time-basic sea-going investigation applications, for example, forecast of seaquakes.

\subsection{Integrity}

It guarantees that data has not been modified by any enemy. $\mathrm{Nu}-$ merous Underwater sensor applications for ecological safeguarding, for example, water quality checking, depend on the trustworthiness of data.

\section{Trends of Technologies}

Before focusing on the examinations and progressions made till yet, it is more vital to think about the waves which are utilized as a part of Underwater correspondence. Chiefly, in this area three kinds of waves are centered around with their properties - acoustic, electromagnetic and optical waves.

\subsection{Acoustic Waves:}

These are the waves which are of the sort of longitudinal waves and engender through adiabatic pressure and extension. Along these lines, they have same course of engendering and vibration. These are utilized as the fundamental transporter in Underwater interchanges. As it is known to us that speed of sound in water relies on a few properties of water, for example, temperature, saltiness, weight and this is the factor which assumes an essential part in correspondence. There is each other idea of retention in these waves. There might be an adjustment in wave vitality while voyaging so this can be changed over to warm because of inelasticity which functions as defect.

\subsection{Electromagnetic Waves}

These are the waves which have electric field and attractive field parts which are opposite to each other and furthermore to the heading of engendering of wave. Ordinarily, electromagnetic waves go with the speed of light. These are organized arranged by recurrence or wavelength in a range called electromagnetic range. It is identified with the hypothesis of electrodynamics and properties of superposition are taken after. It shows both wave and mole- 
cule properties according to wave molecule duality. The vitality is saved when light is assimilated in spite of the fact that it isn't demonstrating molecule yet quantum nature as portrayed in quantum electrodynamics.

\subsection{Optical Waves}

These are the light waves utilized for correspondence. They have enormous preferred standpoint of high information rate however in Underwater it has a few impediments. These are promptly consumed by water and scrambling is additionally conspicuous. By and by they have given better correspondence up to a constrained range. It has more speed than acoustic and along these lines better to use. Additionally, the utilization of different waves could prompt high idleness and could influence marine life that isn't conceivable with optical waves. Along these lines for different Underwater applications optical waves are favored.

\subsection{Study of Experimental Works}

The principle mean to plan Underwater correspondence is to have an effective and dependable system .There is different focal points of Underwater correspondence in this day and age. It isn't another idea to investigate the sea yet in 1912 and significantly prior when titanic sank and arrangement of distinguishing U-water crafts created. The Underwater information connections can be joined with satellite connections for ongoing tasks. These can be utilized for calamity administration process. Along these lines the transmitter and collector for Underwater are like Free Space Optics (FSO) interface in earthbound environment however the main distinction is with the wavelength of task. In spite of the fact that there are various advantages of utilizing Underwater interchanges yet there are a few disadvantages moreover. The primary inconvenience is the battery control required. The data transmission accessible is additionally restricted and the bit mistake rate is high [4]. There is more multipath blurring and spread postponement; the cost is likewise high. They are more inclined to disappointment of framework than earthbound framework. The contrast amongst earthly and Underwater correspondence is clarified in table 1.

Table 1. Comparison between terrestrial and underwater communications

\begin{tabular}{|c|c|c|}
\hline Specification & Underwater & Terrestrial \\
\hline Cost & More & Less \\
\hline Deployment & More sparse & Less sparse \\
\hline Power needed & High & Less \\
\hline Spatial correlation & $\begin{array}{l}\text { Uncorrelated due to } \\
\text { mobility of sensors }\end{array}$ & Correlated \\
\hline
\end{tabular}

In spite of the considerable number of impediments we have better future in Underwater correspondence because of greater security and no climate influence unsettling influence dissimilar to haze or exhaust cloud in earthbound correspondence.

\section{Routing Protocols and Synchronization}

Additionally if there is an immediate connection between source hub and the objective hub; another arrangement of utilizing Transmission Control Protocol (TCP) over a connection which is regulated by a USB CP2102UART is a superior arrangement. Since the transmission capacity is constrained in Underwater correspondence framework. So utilizing optical connections with TCP would even open the ways to video transmission too. For this, communicate MAC is utilized and it gives better outcome. Another idea in Underwater correspondence is the time synchronization which requires the best possible data between two imparting hubs. In any case, because of change in temperature and weight, this factor shifts. When it is accomplished the rate of sending and accepting data can be controlled and the dependability would be more. There are various calculations that can be utilized to have a superior planning synchronization like most extreme probability in light of cyclic prefix, image synchronization in view of Linear Frequency Modulation (LFM) and synchronization in view of pseudo irregular clamor succession. A ten way blurring channel is taken and un-coded Bit Error Rate (BER) is utilized to figure execution with OFDM. It is watched that execution of LFM is superior to others. Also it can oversee deferral and blurring accordingly giving better outcomes to OFDM Underwater correspondence. Notwithstanding it, a versatile pilot coarse planning synchronization strategy is utilized. It uses Constant Amplitude and Zero Auto Correction (CAZAC) succession which has level recurrence reaction. To make the execution of OFDM Underwater framework more solid by utilizing brush compose pilot with CAZAC preface synchronization technique. It enhances exactness and the securing time. The arrangement of OFDM with Underwater correspondence framework on FPGA has been done and the outcomes are noted. Another technique for recurrence jumping is additionally great in Underwater correspondence framework at multipath blurring and solid commotion conditions. So general it has better reenactment comes about for multipoint correspondence. A strategy for Pulse Position Modulation (PPM) has additionally been presented which has enhanced the synchronization complexities and expanded the proficiency. Since high vitality laser is required accordingly xenon light with balanced $\mathrm{Q}$ laser is utilized as a part of the investigation as portrayed in fig. This test is more solid than with modulators.

The flag goes through medium starting with one hub then onto the next, in this way there ought to be legitimate arrangement amongst accepting and transmitting hubs generally the loss of information could be there. Subsequently, high directivity is the primary prerequisite however it isn't conceivable while making associations with AUVs and ROVs. On the off chance that the FOV is little then misalignment issues are more unconventional. Along these lines embeddings the focal point and filtering mirrors can illuminate the reason however at the gathering, the FOV turns out to be more delicate so gives restricting execution. A variety of LEDs at the transmitter side and photodiode cluster at the beneficiary side gives great outcomes which likewise help in the arrangement of transmitter and recipient by checking or shaft guiding. There are different measures which should be considered while setting up interface between the hubs. A model of forecast i.e. testing based model prescient control is proposed for control and following. In AUVs which discovers way out of nearby minima as portrayed.

On the off chance that the physical layer is used at that point better correspondence could be accomplished till $100 \mathrm{~m}$ yet multipath proliferation and Doppler spread is dependably there. In information connect layer, different various access plans can be utilized yet it is watched that lone CDMA worth in giving outcomes yet the dormancy issue stays there. The system layer is a rising technique in which just the utilization of geological steering is helpful rather than proactive and directing conventions however there is an issue of vulnerability in it. In transport layer, the utilization of Event to Sink Reliable Transport (ESRT) is utilized as an instrument to monitor vitality.

\subsection{Secure Routing}

Directing is fundamental for bundle conveyance in UWCNs. For instance, the Distributed Underwater Clustering Scheme (DUCS) does not utilize flooding and limits the proactive steering message trade. Steering is exceptionally testing in UWCNs because of the substantial spread deferrals, the low data transfer capacity, the troubles of battery refills of Underwater sensors, and the dynamic topologies. Along these lines, steering convention ought to be intended to be vitality mindful, hearty, versatile and versatile. Numerous steering conventions have been proposed for Underwater remote sensor organize .However, none of them has been planned with security as an objective. Directing assaults can handicap the whole system's activity. Caricaturing, changing, or replaying directing data influences steering. 


\section{Conclusion}

This paper gives the general perspective of the need of Underwater remote correspondence and its applications. Notwithstanding much improvement around there of the Underwater remote correspondence, there is as yet a massive extension so more research as real piece of the sea base yet stays unexploded. Underwater Sensor Networks is an exceptionally late innovation that tries to take after similar strides than earthly remote systems in an altogether different and testing system condition. There is an expanding enthusiasm for USWN advances and their potential applications. Underlining the particular attributes of these systems, conceivable assaults, and counter measures. The fundamental research challenges identified with secure time synchronization, confinement, and directing have additionally been overviewed. The examination issues stay totally open for future examination, and locate the best method is extend free appropriated situating plan since its give the substantial scale range and range free strategy at show time .

\section{Future Scope}

We do have are great purposes behind $4 \mathrm{G}$ improvement and an assortment of current and developing advancements to make $4 \mathrm{G}$ a reality. Featuring the essential drivers for $4 \mathrm{G}$ remote frameworks are taken a toll, speed, adaptability, and widespread access. Both specialist co-ops and clients need to diminish the cost of remote frameworks and the cost of remote administrations. The more affordable the cost of the framework, the more individuals who will need to claim it.

The high data transfer capacity prerequisites of up and coming spilling video requires an adjustment in the plan of action the specialist organizations use-from the committed channel per client model to one of a mutual use, as-parcels seem to be required model. This will undoubtedly be the model specialist co-op's utilization when $4 \mathrm{G}$ frameworks are ordinary (if not previously).

\section{References}

[1] D. Naresh Kumar, "Implementation for wideband applications using UWB fractal patch antenna", AIP Conference Proceedings, Volume 1952, Issue 1, April 2018.

[2] Ian F. Akyildiz , Dario Pompili, Tommasomelodia, "Underwater Acoustic Sensor Networks: Research Challenges", Broadband And Wireless Networking Laboratory, School Of Electrical And Computer Engineering, Georgia Institute Of Technology, Atlanta, Ga 30332, Usa.

[3] D. Naresh Kumar, "Adaptive Bandwidth Allocation in Wireless Networks with Multilevel Degradable Quality of Service", International Journal of Research in Science and Engineering, Vol. 2, Issue 1, pp. 17-21, August 2015.

[4] D. Naresh Kumar, "Performance Analysis of Modified Round Robin Algorithm", Journal of Chemical and Pharmaceutical Sciences, pp. 39-42, June 2017.

[5] Weichao Wang, Jiejun Kong, "Visualisation Of Wormholes In Underwater Sensor Networks: A Distributed Approach", Int. J. Security And Networks, Vol. 3, No. 1, 2008.

[6] "Securing Underwater Wireless Communication Networks", Electrical And Electronics Engg. Seminar Report 2013.

[7] Ashvini P. And Sivasankaran V., "Securing Underwater Wireless Communication Networks-Literature Review", Arpn Journal Of Engineering And Applied Sciences .C2006-2015 Asian Research Publishing Network (Arpn). All Rights Reserved.

[8] Suraj S. Kasture, Nikhil Gudpelliwar, "Securing Underwater Wireless Communication Networks-Literature Review", Ashvini P. And Sivasankaran V. Department Of Electronics And Communication System, Arunai College Of Engineering, Thiruvannamalai, India. 